\title{
THE EFFECT OF THE DISPERSION OF REWARDING AND REWARDLESS FLOWERS ON VISITATION AND CONSTANCY BY BUMBLEBEES (BOMBUS
} IMPATIENS)

\author{
Emma Katz ${ }^{1 *}$ and Carla J. Essenberg ${ }^{1,2}$ \\ ${ }^{1}$ Bates College, Program in Environmental Studies, Bates College, Lewiston, ME 04240, USA \\ ${ }^{2}$ Department of Biology, Faculty of Science, Bates College, Lewiston, ME 04240, USA
}

\begin{abstract}
Deceptive pollination strategies, in which plants rely on animal pollinators but provide no benefits to their pollinators, have evolved many times in angiosperms. However, the conditions that favour deceptive pollination strategies over mutualistic strategies are poorly understood. One factor that may be important for the success of deceptive strategies is the plants' dispersion in relation to co-flowering, rewarding species. We compared pollinator behaviour across two artificial environments, one in which a rewardless species was mixed with a rewarding species and one in which the two species occurred in contiguous patches. Bumblebees both encountered and visited rewardless flowers more often when they were mixed with rewarding species. However, the rate of switching was also higher under those conditions, which could result in higher rates of interspecific pollen transfer. The environmental conditions most favourable to deceptive pollination strategies may vary depending on the vulnerability of the plant species to interspecific pollen transfer.
\end{abstract}

Keywords: Generalized food deception; magnet species; aggregation; facilitation; competition; foraging behaviour

\section{INTRODUCTION}

Deceptive pollination, a strategy in which plants provide no reward to their pollinators but still rely on pollinator visits, has evolved independently many times in the angiosperms, and reversion from rewardlessness to mutualism has also occurred multiple times (Renner 2006; Smithson 2009). An active area of research focuses on the fitness costs and benefits of deceptive strategies (Jersáková et al. 2006), but why deception evolves in some species while others evolve mutualistic strategies has received less attention. Biotic environmental conditions may be important in determining the success of deceptive strategies, as deceptive species rely on heterospecific flowers or other biological conditions to keep pollinators in their vicinity (Renner 2006; Duffy \& Johnson 2017). Probably the most common deceptive strategy, known as generalized food deception, is to present flowers that offer signals typical of rewarding plant species without mimicking any particular model (Jersáková et al. 2006). Whether generalized food deception is favoured over mutualism may depend on the abundance of co-flowering, rewarding species as well as the dispersion of rewarding and rewardless plants (Lammi \& Kuitunen 1995; Johnson et al. 2003; Internicola et al. 2006; Juillet et al. 2007).

Received I7 March 2018, accepted 2I June 2018

*Corresponding author: emmajkatzI@gmail.com
Previous studies suggest that the degree of mixing between rewarding and rewardless plant species can affect pollinator visitation rates to the rewardless species, how often pollinators switch between species, and the rewardless species' reproductive output (Keasar 2000; Internicola et al. 2006; Internicola et al. 2007; also see Hanoteaux et al. 2013; Geslin et al. 20I4; de Waal et al. 20I5; Bruckman \& Campbell 20I6, which consider effects of dispersion on pollinator-mediated interactions between rewarding plant species). However, the nature of these effects varies across studies, suggesting that multiple aspects of dispersion influence rewardless species' success (Tab. I). An experiment manipulating both the degree of mixing and density of the rewardless orchid Dactylorhiza sambucina and a dissimilar rewarding species, Muscari neglectum, found that increasing aggregation of both species negatively influenced the reproductive success of the rewardless plant (Internicola et al. 2006). In contrast, Keasar (2000) found that rewardless artificial flowers received more visits and switching between species was less common when rewarding and rewardless flowers were separated in spatially distinct, monospecific patches than when they were mixed within a single, large patch, suggesting that aggregation may sometimes be favourable to rewardless species. One explanation for the relatively high visitation to rewardless flowers in Keasar's separated treatment is that the distance between the monospecific patches made switching away from the rewardless species more costly than it was when both species occurred in a single patch, favouring strings of visits to the rewardless species each time a rewardless patch was visited. Consistent with this idea are the contrasting results of 
Table I. Summary of previous studies exploring effects of dispersion of rewarding and rewardless flowers on aspects of the rewardless flowers' pollination success. The posited mechanism explaining each result is our interpretation and may not reflect the original researchers' text.

\begin{tabular}{|c|c|c|c|}
\hline Citation & Manipulated Variables & $\begin{array}{l}\text { Effect of Aggregation on } \\
\text { rewardless flowers }\end{array}$ & Posited Mechanism \\
\hline Internicola et al. 2006 & $\begin{array}{l}\text { Degree of mixing and density of } \\
\text { rewardless and rewarding species }\end{array}$ & Lower reproductive success & $\begin{array}{l}\text { Travel cost of switching distant } \\
\text { patches favoured thorough } \\
\text { sampling in both treatments }\end{array}$ \\
\hline Keasar (2000) & $\begin{array}{l}\text { Intermixed vs clustered, spatially } \\
\text { separated patches }\end{array}$ & $\begin{array}{l}\text { Increased visits, decreased } \\
\text { switching }\end{array}$ & $\begin{array}{l}\text { Distance between monospecific } \\
\text { patches made switching costly }\end{array}$ \\
\hline Internicola et al. (2007) & $\begin{array}{l}\text { Mixed vs monospecific patches, } \\
\text { both treatments spatially } \\
\text { separated }\end{array}$ & No significant effect & $\begin{array}{l}\text { Travel cost of switching patches } \\
\text { favoured thorough sampling in } \\
\text { both treatments }\end{array}$ \\
\hline
\end{tabular}

Internicola et al. (2007), who compared bee behaviour in mixed versus monospecific flower patches, with patches in both treatments spatially separated. In the Internicola et al. (2007) study, mixing had no significant effect on visitation to the rewardless species, perhaps because travel costs associated with moving to a new patch favoured relatively thorough sampling of rewardless flowers in both mixed and monospecific patches.

These results leave open the question of how mixing versus separation of rewarding and rewardless species affects pollinator behaviour when there are no between-patch travel costs. Rewardless flowers may actually receive more visits when mixed with rewarding flowers than when rewarding and rewardless flowers occur in contiguous, pure patches. In both situations, the cost of switching away from the rewardless species should be small, but pollinators would likely encounter the rewardless flowers more frequently when they were mixed with rewarding flowers than when they occurred in monospecific zones of the flower patch.

The aim of the current study was to examine the effect of the mixing of rewarding and rewardless flowers within a continuous patch on visitation rates to the rewardless species as well as the frequency of switching between species. We observed bumblebees (Bombus impatiens) foraging on arrays of artificial flowers in which rewarding and rewardless species had similar, but distinguishable colours. This experimental context modelled a deceptive species with imperfect mimicry of a co-flowering, rewarding species.

\section{MATERIALS AND METHODS}

We observed the effect of the dispersion of rewarding and rewardless artificial flowers on the foraging behaviour of bumblebees (Bombus impatiens). The two flower "species" differed in colour so that bees could distinguish them, although the colours were similar enough that bees may have needed to learn to discriminate between them. Prior to experimental trials, bees were given experience visiting the rewarding flower type, so our experiment modelled a situation in which bees were only naive to the rewardless species. In each experimental trial, a bee was randomly assigned to one of two treatments: a floral array in which the rewarding and rewardless flowers formed contiguous patches ("separated") or an array in which the two flower types were mixed together ("mixed"). Each experimental trial was split into two parts, with each part consisting of I5 flower visits. Analyzing these two parts of the experimental trial separately allowed us to assess how bee behaviour changed with experience. We recorded both the number of visits to each flower type and the number of switches between flower types.

\section{Bumble bee husbandry}

We used commercially-reared bumble bees (Bombus impatiens, Koppert Biological Systems) for this experiment. Bees were given pollen within the colony box and 20\% sucrose solution $(w / w)$ from a feeder in a foraging arena. The data we present in this paper came from a total of $4 \mathrm{I}$ individual foragers from three Bombus impatiens colonies (16, I4, and II foragers from each).

\section{Artificial flowers and experimental chamber}

Artificial flowers consisted of a small reservoir made from the cap of a I mL microcentrifuge tube surrounded by a coloured paper corolla $(3.5 \mathrm{~cm}$ diameter $)$ reinforced with a plastic ring positioned underneath it (Fig. I). Each flower sat

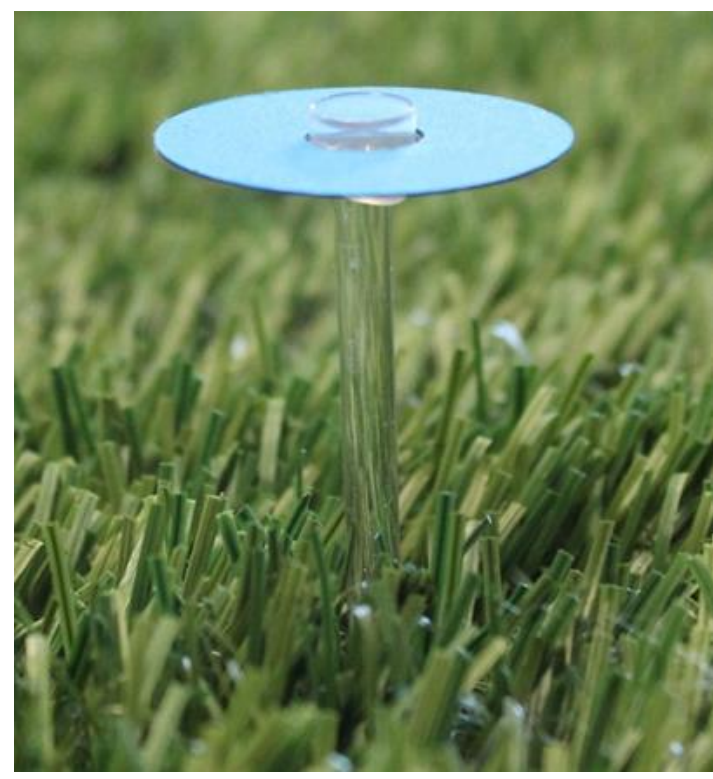

FIGURE I. Artificial flower used in the experiment. 


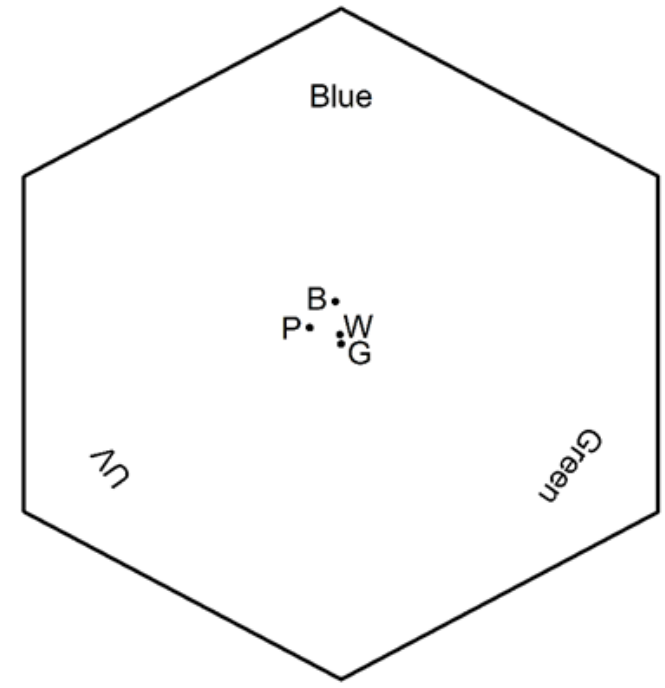

Figure 2. Colours of artificial flowers in $B$. impatiens colour space. $\mathrm{W}=$ white, $\mathrm{B}=$ blue, and $\mathrm{P}=$ purple corolla. $\mathrm{G}=$ average colour of the green turf background. The diagram was made according to Chittka (1992) using data on the spectral sensitivities of B. impatiens receptors from Skorupski and Chittka (2010).

on top of a clear plastic rod that served as a stalk. Flowers with blue corollas contained sucrose rewards, located in the center of the flower, while flowers with purple corollas were empty. Positions of the corolla colours in B. impatiens colour space are shown in Fig. 2 (Chittka 1992; Skorupski \& Chittka 20I0). The blue and purple colours used were relatively similar: their chromatic contrast ( $D=0.1059$ units) was great enough to be distinguishable to bees but with reduced accuracy compared to more highly contrasting colours (Dyer \& Chittka 2004). Therefore, bees should have been capable of distinguishing the two flower types but may have needed to learn to discriminate between them. Our experiment, therefore, is based on a situation in which the rewardless species is an imperfect mimic of the rewarding species.

Both the training and experimental trials took place inside an experimental chamber (dimensions: $70 \mathrm{~cm}$ long $\times 60 \mathrm{~cm}$ wide $\times 50 \mathrm{~cm}$ tall), the floor of which was covered with artificial turf. Holes to accommodate flower stalks were drilled into a board forming the floor of the chamber in an 8 $\times 8$ array with $6 \mathrm{~cm}$ spacing between holes.

\section{Training Sessions}

Prior to individual experimental trials, foragers from each colony were given opportunities to visit white artificial flowers (which match the colour of the maintenance feeder) and then blue flowers. Training sessions took place several times a day during the 3-5 days prior to the beginning of experimental trials for each colony and then were repeated periodically throughout the experiment. Flowers had white corollas for the first I-2 days, until several foragers learned to use the flowers. We used blue corollas for all subsequent training sessions. Prior to each training session, six flowers were placed in randomly-chosen locations in the $8 \times 8$ array and each flower was filled with $20 \mu 150 \%$ sucrose solution $(w / w)$. The entire colony was allowed to forage on the training array for 30 minutes, and flowers were refilled as they emptied. We recorded the identities of bees foraging on the flowers during each training session.

\section{Experimental trials}

Foragers were selected for experimental trials and assigned to treatments as follows. Only bees that had been observed foraging on artificial flowers during the training sessions were used. Of those bees, we selected the first bee to emerge from the colony box after the chamber was prepared for an experimental trial. After a bee was selected, it was randomly assigned to either the separated or mixed treatment. We recorded $2 \mathrm{I}$ bees in the separated treatment and 20 bees in the mixed treatment

In both treatments, the experimental array consisted of 24 blue (rewarding) flowers and 24 purple (unrewarding) flowers arranged in a continuous patch $(45.5 \mathrm{~cm} \times 45.5 \mathrm{~cm})$. Each blue flower contained I $\mu$ l of $50 \%$ sucrose solution and each purple flower was empty. Equal numbers of flowers were located within each quarter of the $8 \times 8$ array, with their locations chosen at random. In the mixed treatment, flowers of both colours were randomly dispersed across the entire array. In the separated treatment, each quarter of the array contained flowers of one type only (i.e., blue or purple), with neighbouring quarters containing different flower colours (Fig. 3). Randomizations of flower locations were generated using the sample $($ ) command in $\mathrm{R}$ version 3.3.0 ( $\mathrm{R}$ Core Team 20I4).

Each experimental trial was split into two parts. After the bee had probed the reservoirs of I 5 different flowers, the light was turned off, all blue flowers that had been visited were refilled, and locations of rewarding and rewardless flowers and zones were re-assigned. The bee was then released to probe another 15 flowers. Flowers were not refilled while the bee was foraging. Revisits to flowers did not count towards the I5 visits. Therefore, the number of flowers probed corresponds to the number that could have been pollinated, had these been real flowers. The division of the experiment into two parts allowed us to compare the bee's initial, naive responses to the rewardless flowers with its responses after limited experience with the rewardless flowers. Making this comparison without taking a break to rearrange and refill the flowers would have been complicated by the bees' tendency to revisit flowers.

Experimental trials were recorded using a video camera, which was located above and just to one side of the array. All behavioural data were acquired by transcribing these video recordings. Two bees were excluded from subsequent analysis because the camera stopped recording very early in one trial. In three other trials, we failed to videotape a full I5 flower visits (not counting revisits) because of a camera failure or other error. We used only the section of the trial that we had on video in our analyses (constituting 7, I3, and 13 flower visits for the three trials).

\section{Data Analysis}

We analysed the effects of treatment (separated or mixed) and trial part ( $\mathrm{I}$ or 2 ) on several response variables. To provide information about how floral dispersion would be likely to influence plant pollination success, we analysed the proportion of the bee's flower visits, excluding revisits, that 
a. Mixed

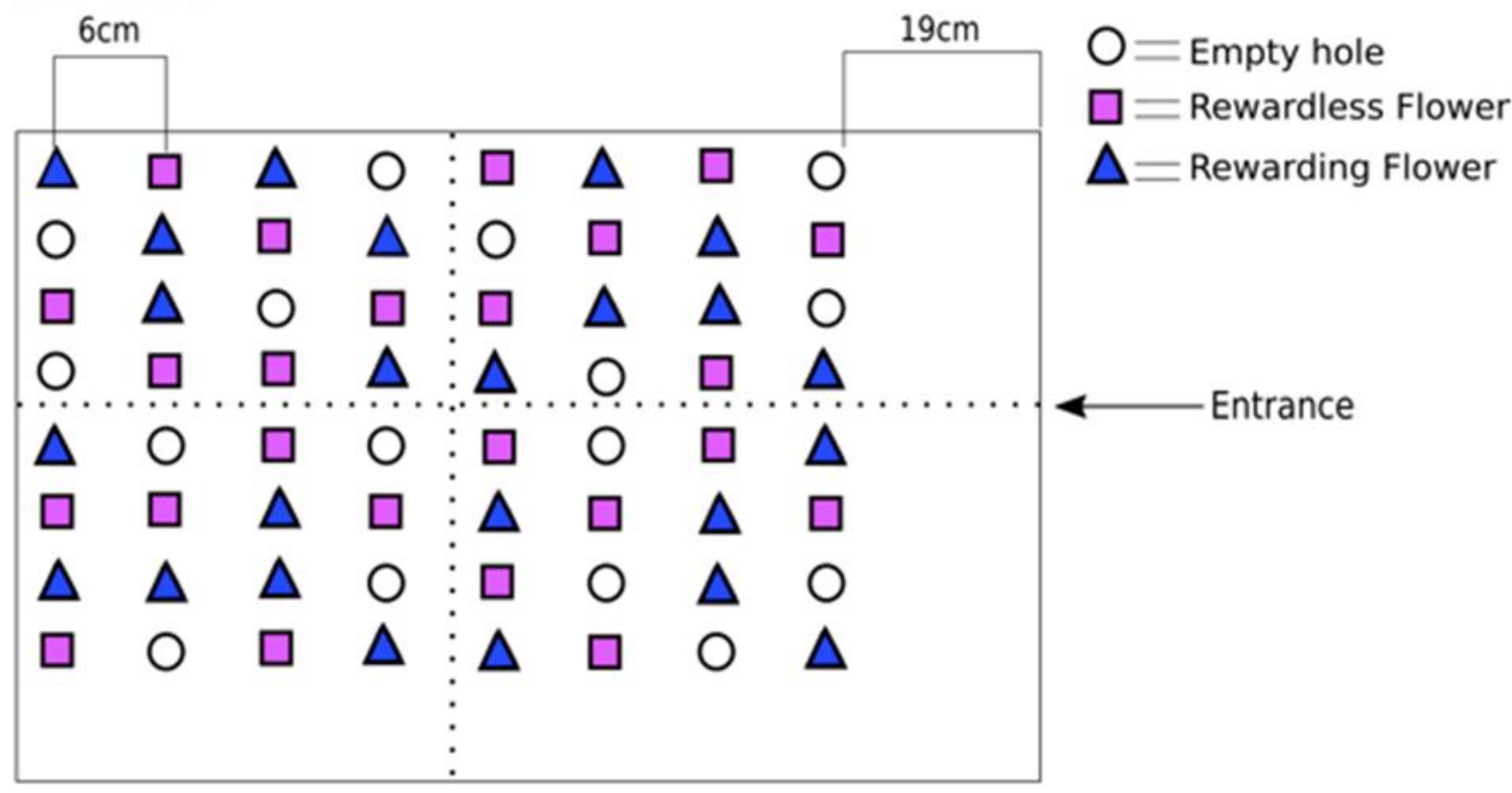

\section{b. Separated}

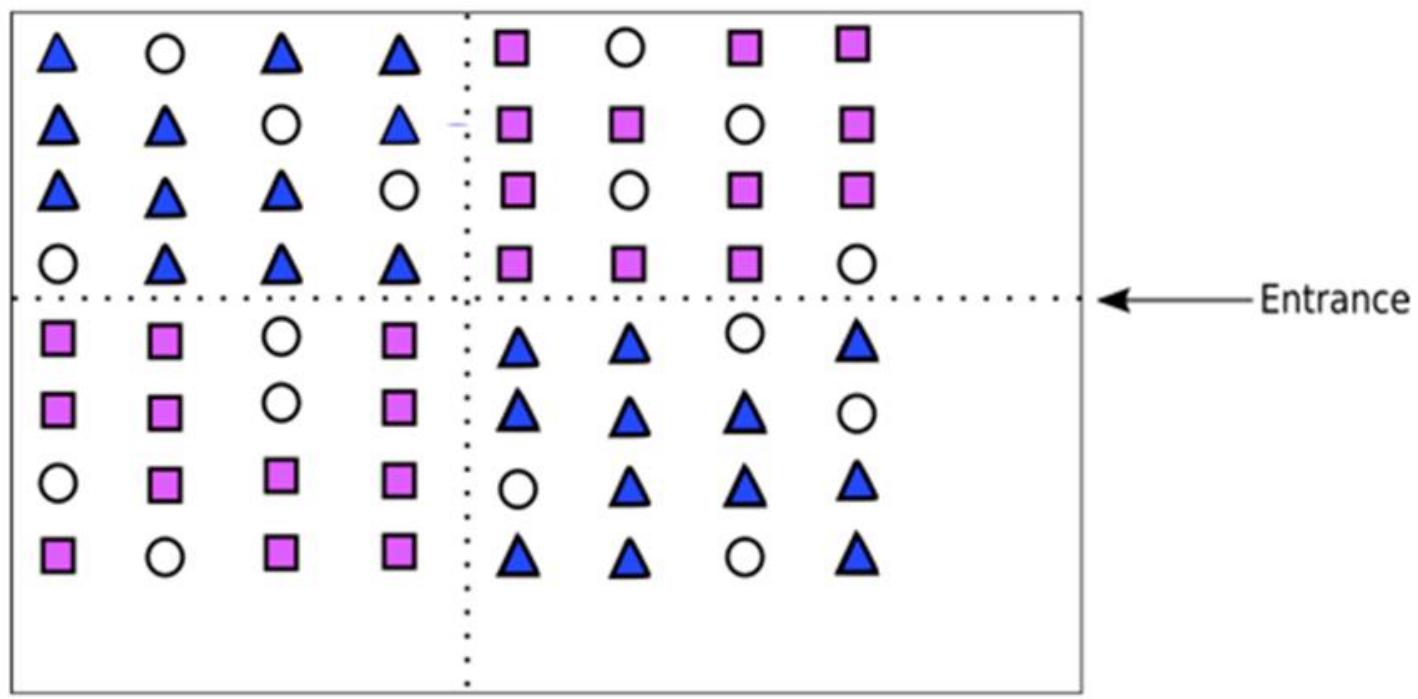

FIGURE 3. Example of spatial arrangements of rewarding and rewardless flowers in the mixed (a) and separated (b) treatments.

were to the rewardless flower type, and the proportion of flower to flower transitions in the bee's visit sequence (including revisits) in which the bee switched flower colours. We counted as visits only occasions when the bee both landed on and came in contact with the centre of the flower (i.e., its head crossed at least part of the reservoir in the flower's centre) because those were occasions when pollination could have occurred, were these real flowers.

To provide further insight into the behavioural mechanisms underlying patterns we observed in flower visitation, we also analysed the proportion of flower encounters that were to the rewardless flower type, excluding encounters of previously visited flowers. A flower encounter was defined as any occasion on which the bee flew within I $\mathrm{cm}$ of the edge of the corolla, as seen from above, whether it landed or not. We did not count as encounters occasions when the bee was flying near the top of the chamber rather than close to the flowers or occasions when the bee flew backwards towards a flower, unless she ended up directly over the flower. Bees sometimes flew over the array for extended periods without landing. We were interested in occasions when the bee was actively searching for a flower to visit and therefore counted only the last five flower encounters before the bee landed on a flower.

In analyzing proportions of flower visits and encounters that were to the rewardless flower type, we used linear, mixed 
effects models in which treatment, trial part, and their interaction were treated as fixed effects. Bee ID nested within colony were treated as random effects; including Bee ID as a random effects factor allowed us to compare parts $I$ and 2 of each bee's trial.

We also wished to know whether the bees discriminated against rewardless flowers and, if so, whether they discriminated more strongly as they gained experience. Therefore, we analysed the effects of flower type, treatment (separated or mixed), trial part (I or 2), and all interactions on the proportion of encounters of each flower type that resulted in the bee visiting the flower. We considered only encounters of flowers that the bee had not visited previously. As before, we used a linear, mixed effects model, with bee ID nested within colony included as random effects.

In all of these analyses, interaction terms were dropped from the model if nonsignificant. Proportion of flower visits was log-transformed to improve fit to the normal distribution. After this transformation, residuals from all of the tests met the assumptions of normality and homoscedasticity. Analyses were carried out using the $\operatorname{lmer}()$ function in the lmerTest package in $\mathrm{R}$ version 3.4.I (Kuznetsova et al. 20I5; R Core Team 2017).

\section{RESULTS AND DISCUSSION}

Foraging bumblebees (Bombus impatiens) made a higher proportion of their visits to rewardless flowers when they were mixed with rewarding flowers than when rewarding and rewardless flowers formed contiguous patches (Fig. 4a, Tab. 2). We excluded revisits because of what we perceived as an unnaturally high revisitation rate: $15 \%$ of the bees' visits to rewardless flowers and $33 \%$ of their visits to rewarding flowers were revisits. A similar treatment effect was observed if revisits were included.

Two different mechanisms could explain a lower rate of visits to rewardless flowers when the two flower types formed single-species patches than when they were mixed together. First, bees could simply encounter rewardless flowers less frequently when the two species occurred in separate patches than when they were mixed with rewarding flowers. Arearestricted search behaviour, in which foragers turned more frequently and made shorter flights after encountering rewards than after unrewarding visits, could allow bees to do a larger proportion of their searching in rewarding than rewardless patches, even if they had no preference for the rewarding species (Pyke 1978; Waddington I980; Burns \& Thomson
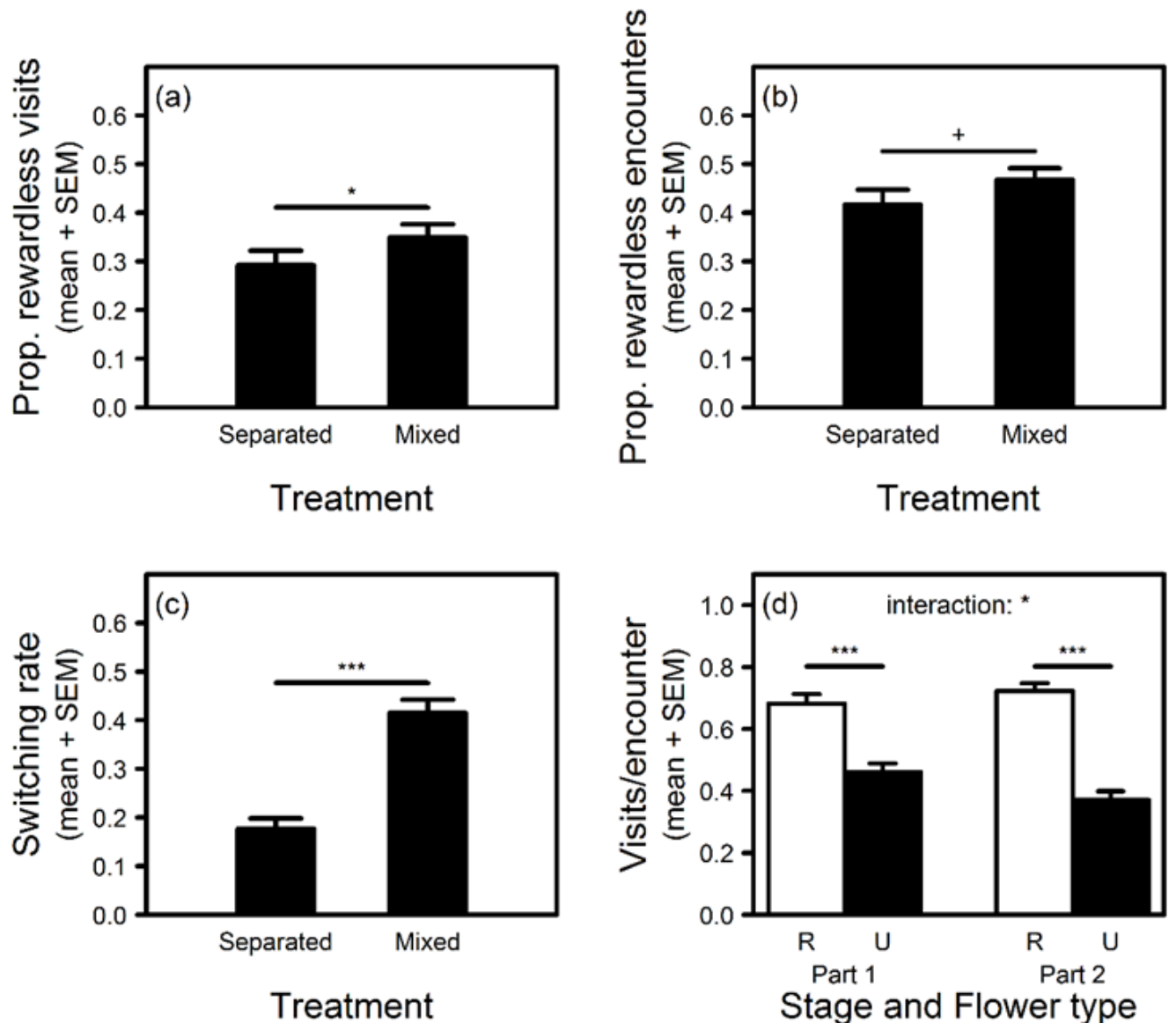

FIGURE 4. Effects of flower dispersion or forager experience on the proportion of bumblebee (B. impatiens) flower (a) visits and (b) encounters that were to the rewardless (purple) artificial flower type, (c) proportion of transitions that were switches between flower types, and (d) proportion of encounters to rewardless ("U", white bars) and rewarding ("R", black bars) flowers that resulted in visits. Examples of the two dispersion treatments are shown in Fig. 3. Each part of each experimental trial consisted of I5 flower visits, not including revisits. Revisits are omitted except in panel (c) Results of statistical analyses are provided in Tab. 2. $+P<0 . \mathrm{I},{ }^{*} P<0.05,{ }^{*} P<0.0 \mathrm{I}$, $P<0.00 \mathrm{I}$. 
TABLE 2. Effects of dispersion of rewarding and rewardless flowers on the relative frequency of bumblebees' encounters of and visits to rewardless flowers, frequency of switches between flower types, and tendency to visit encountered flowers of each type. Dispersion treatments were separated or mixed, as shown in Fig. 3. Part refers to the first vs. second part of the experimental trial. Flowers that had been visited previously were excluded from analysis of all variables except switching frequency. Interactions are shown where significant; all omitted interactions have $p>0.35$ Results are from mixed effects models, with bee identity nested within colony as random effects. Bee identity did not explain any variation in proportion of visits or encounters to rewardless flowers, and colony did not explain any variation in the proportion of encountered flowers that were visited. Proportion of visits to rewardless flowers was log-transformed to improve normality. Data are from 2I bees in the separated treatment and 20 bees in the mixed treatment, from 3 colonies.

\begin{tabular}{|c|c|c|c|c|}
\hline & Factor & Den. df & $F$ & $P$ \\
\hline \multirow[t]{2}{*}{ Proportion of bee's visits that are to rewardless flowers } & Dispersion treatment & 77.4 & 4.3 & 0.042 \\
\hline & Part & 76.8 & $2 . \mathrm{I}$ & 0.152 \\
\hline \multirow[t]{2}{*}{ Proportion of encountered flowers that are rewardless } & Dispersion treatment & 77.4 & 3.9 & 0.053 \\
\hline & Part & 76.8 & 0.5 & 0.488 \\
\hline \multirow{2}{*}{$\begin{array}{l}\text { Proportion of transitions that are switches between flower } \\
\text { types }\end{array}$} & Dispersion treatment & 42.5 & 95.9 & $<0.001$ \\
\hline & Part & 46.7 & 0.2 & 0.646 \\
\hline \multirow[t]{5}{*}{ Proportion of encounters that resulted in a visit } & Flower type & I I 9.3 & 132.3 & $<0.001$ \\
\hline & Dispersion treatment & 37.I & O.I & 0.762 \\
\hline & Part & II 9.3 & I.I & 0.302 \\
\hline & Treatment $\times$ Part & II 9.3 & 3.5 & 0.065 \\
\hline & Flower type $\times$ Part & 45.4 & 6.9 & 0.010 \\
\hline
\end{tabular}

2006). If the species were mixed together randomly, however, this tactic would be ineffective. Waddington (I980) demonstrated this consequence of flower dispersion with honeybees, which made higher proportions of rewarding visits when rewarding flowers were clumped than when they were distributed randomly amongst unrewarding flowers with identical cues.

Consistent with predictions generated by area-restricted models of search behaviour and previous findings in honey bees, bees encountered a higher proportion of rewardless flowers when rewarding and rewardless flowers were mixed together than when they were separated into contiguous patches (again, excluding previously visited flowers), although the difference was only marginally significant $(P=0.053$, Fig. 4 b, Tab. 2).

A second reason for rewardless plants to receive more visits in our mixed treatment than in the separated treatment could have been that bees were more willing to sample a second flower species when flowers of the rewarding species were spaced farther apart, on average, forcing the bees to make longer flights between neighbouring conspecific flowers. Foragers, including bees, do often broaden their diets in response to a low density of high-quality food resources (Pyke 1984; Kunin \& Iwasa 1996; Gegear \& Thomson 2004; Ishii 2005). However, we found no evidence of decreased preference for the rewarding species in the separated treatment compared to the mixed treatment. Comparison of the proportion of flower encounters that resulted in flower visits (excluding revisits) revealed a significantly higher tendency to visit the rewarding than the unrewarding type in both dispersion treatments (Fig. 4d, Tab. 2). Treatment did not influence the strength of this difference in visit tendency.

Our results contrast with those of Keasar (2000), who found that bees visited rewardless flowers more often when they were arranged in distinct patches, with space in between them, than when mixed together with rewarding flowers. This difference in outcome may be because the higher energy cost of traveling $80-120 \mathrm{~cm}$ between patches in Keasar's separated treatment led bees to remain in the rewardless patches longer than they did in our experiment, in which the patches were contiguous. If so, then the spatial scale at which plant species are associated with each other could be critical in determining whether being intermixed with rewarding plants is favourable to a rewardless strategy. In plant communities composed of small individuals occurring in close proximity (e.g., $6 \mathrm{~cm}$ from nearest neighbours, as in our experiment), intermixing might be favourable for rewardless species, whereas aggregated distributions would be more favourable when individuals were spaced farther apart.

The spatial arrangement of flowers also had a significant effect on the frequency of switches between rewarding and rewardless flowers, with a higher rate of switching between flower types occurring in the mixed treatment than in the separated treatment (Fig. 4c, Tab. 2). Furthermore, the magnitude of this increase in the rate of between-species switches was considerably greater than the relatively small increase in visitation experienced by the rewardless flower type in the mixed treatment. Keasar (2000) and Internicola et al. (2007) also found that the total number of shifts between rewarding and rewardless flowers was significantly higher when the two were mixed. Higher rates of switching between species can increase interspecific pollen transfer, which is typically detrimental to both plant species (Rathcke I983; Brown \& Mitchell 200I; Morales \& Traveset 2008). However, the degree to which pollinator inconstancy reduces pollination success varies across plant taxa. For instance, the packaging of pollen into pollinaria in many orchid species allows their pollen to be deposited on conspecific stigmas even after many intervening visits to other species' flowers 
(Jersáková et al. 2006). As a result, rewardless orchids may be able to take advantage of increased visitation rates experienced when growing mixed together with rewarding species while experiencing little ill effect from the frequent switching between species that pollinators exhibit under those conditions. This advantage could help to explain the high prevalence of food deceptive pollination systems in the Orchidaceae.

Bees' preference for the rewarding flower type increased over the course of each trial: the difference in number of visits per flower encounter for rewarding and rewardless flowers was greatest in the second part of each trial (Fig. 4d, Tab. 2). The magnitude of this shift was similar across the two dispersion treatments. These results suggest that avoidance learning occurred in both treatments and that dispersion had little, if any, effect on learning rate. Similarly, Internicola et al. (2007) found that over time bumblebees visited fewer rewardless flowers regardless of whether the flowers occurred in mixed or homogeneous patches. In our experiment, however, the increased preference for the rewarding flower type did not translate into a significant decrease in visitation rate to the rewardless flowers over the course of an experimental trial (Tab. 2).

Our results contrast with those of previous studies in suggesting that growing mixed with a co-flowering, rewarding species can increase the quantity of visits a rewardless species experiences. However, being mixed with another species also carries a cost: pollinators switch between species more often when they are mixed, which is likely to reduce the quality of visits the rewardless species experiences. Therefore, which conditions are most favourable to a rewardless strategy will likely vary depending on the plant's susceptibility to interspecific pollen transfer as well as on the number of visits needed to achieve successful pollination.

\section{ACKNOWLEDGEMENTS}

Funding was provided by Bates College. We thank Colin A. McIntire for the artificial flower design and Xiaomeng Wang for other contributions to the methods.

\section{REFERENCES}

Brown BJ, Mitchell RJ (200I) Competition for pollination: effects of pollen of an invasive plant on seed set of a native congener. Oecologia I29:43-49.

Bruckman D, Campbell DR (2016) Pollination of a native plant changes with distance and density of invasive plants in a simulated biological invasion. American Journal of Botany I03:I458-I465.

Burns JG, Thomson JD (2006) A test of spatial memory and movement patterns of bumblebees at multiple spatial and temporal scales. Behavioural Ecology 17:48-55.

Chittka L (1992) The colour hexagon: a chromaticity diagram based on photoreceptor excitations as a generalized representation of colour opponency. Journal of Comparative Physiology A 170:533543.

de Waal C, Anderson B, Ellis AG (2015) Relative density and dispersion pattern of two southern African Asteraceae affect fecundity through heterospecific interference and mate availability, not pollinator visitation rate. Journal of Ecology 103:513-525.
Duffy KJ, Johnson SD (2017) Effects of distance from models on the fitness of floral mimics. Plant Biology I9:438-443.

Dyer AG, Chittka L (2004) Biological significance of distinguishing between similar colours in spectrally variable illumination: bumblebees (Bombus terrestris) as a case study. Journal of Comparative Physiology A 190:I05-II4.

Gegear RJ, Thomson JD (2004) Does the flower constancy of bumble bees reflect foraging economics? Ethology I 10:793-805.

Geslin B, Baude M, Mallard F, Dajoz I (20I4) Effect of local spatial plant distribution and conspecific density on bumble bee foraging behaviour. Ecological Entomology 39:334-342.

Hanoteaux S, Tielbörger K, Seifan M (2013) Effects of spatial patterns on the pollination success of a less attractive species. Oikos I22:867-880

Internicola AI, Juillet N, Smithson A, Gigord LDB (2006) Experimental Investigation of the Effect of Spatial Aggregation on Reproductive Success in a Rewardless Orchid. Oecologia I50:43544I.

Internicola AI, Page PA, Bernasconi G, Gigord LDB (2007) Competition for pollinator visitation between deceptive and rewarding artificial inflorescences: an experimental test of the effects of floral colour similarity and spatial mingling. Functional Ecology 2I:864-872.

Ishii HS (2005) Analysis of bumblebee visitation sequences within single bouts: Implication of the overstrike effect on short-term memory. Behavioral Ecology and Sociobiology 57:599-610.

Jersáková J, Johnson SD, Kindlmann P (2006) Mechanisms and evolution of deceptive pollination in orchids. Biological Reviews of the Cambridge Philosophical Society 8I:219-235.

Johnson SD, Peter CI, Nilsson LA, Agren J (2003) Pollination success in a deceptive orchid is enhanced by co-occurring rewarding magnet plants. Ecology 84:2919-2927.

Juillet N, Gonzalez MA, Page PA, Gigord LDB (2007) Pollination of the European food-deceptive Traunsteinera globosa (Orchidaceae): The importance of nectar-producing neighbouring plants. Plant Systematics and Evolution 265:I23-I29.

Keasar T (2000) The spatial distribution of nonrewarding artificial flowers affects pollinator attraction. Animal Behaviour 60:639. 646.

Kunin W, Iwasa Y (1996) Pollinator foraging strategies in mixed floral arrays: density effects and floral constancy. Theoretical Population Biology 49:232-263.

Kuznetsova A, Brockhoff PB, Christensen RHB (2015) Package 'ImerTest'. R package version 2.

Lammi A, Kuitunen M (1995) Deceptive pollination of Dactylorhiza Incarnate: An experimental test of the magnet species hypothesis. Oecologia I0I:500-503.

Morales CL, Traveset A (2008) Interspecific pollen transfer: magnitude, prevalence and consequences for plant fitness. Critical Reviews in Plant Sciences 27:22I-238.

Pyke GH (1978) Optimal foraging: Movement patterns of bumblebees between inflorescences. Theoretical Population Biology 13:72-98.

Pyke GH (1984) Optimal foraging theory: a critical review. Annual Review of Ecology and Systematics I5:523-575.

R Core Team (2014) R: A language and environment for statistical computing. R Foundation for Statistical Computing, Vienna, Austria.

R Core Team (2017) R: A language and environment for statistical computing. R Foundation for Statistical Computing, Vienna, Austria. 
Rathcke B (1983) Competition and facilitation among plants for pollination. Pollination Biology:305-325.

Renner SS (2006) Rewardless flowers in the angiosperms and the role of insect cognition in their evolution. In: Waser NM, Ollerton J (eds) Plant-pollinator interactions: from specialization to generalization University of Chicago Press, Chicago, pp I23-I44.

Skorupski P, Chittka L (2010) Photoreceptor spectral sensitivity in the bumblebee, Bombus impatiens (Hymenoptera: Apidae). PLoS One 5:eI2049.
Smithson A (2009) A plant's view of cheating in plant-pollinator mutualisms. Israel Journal of Plant Sciences 57:15I-I63.

Waddington KD (1980) Flight patterns of foraging bees relative to density of artificial flowers and distribution of nectar. Oecologia 44:199-204. 